\title{
Effect of recovery time and loading modes on mechanical properties measures in damaged human cortical bone
}

\author{
Won JOO* and Dwight T. DAVY** \\ *Department of Engineering, Robert Morris University \\ 6001 University Blvd. Moon Township, PA, 15108, U.S.A. \\ E-mail: joo@rmu.edu \\ **Department of Mechanical and Aerospace Engineering, Case Western Reserve University \\ 10900 Euclid Avenue, Cleveland, OH, 44106, U.S.A.
}

Received: 17 March 2018; Revised: 7 June 2018; Accepted: 25 September 2018

\begin{abstract}
Skeletal fragility is an important orthopedic concern. In this study, the effect of the recovery period after damage under different mode of damage loading on the human cortical bone was investigated. Human cortical bone samples were damaged with one of three different loading modes (tension, compression and torsion) and the change of mechanical properties (stiffness, viscous relaxation and energy dissipation) before and after the damage with respect to different recovery time $(1,10,30,60$ and 100 minutes) were measured. Results showed that measures of mechanical properties after damage varied significantly with recovery time and load modes. Typically, significant changes in property ratios occurred in the first 10 minutes for loading modulus measures in all loading modes. In addition, results showed that, with respect to loading mode effects, all torsional modulus changes were largest followed by tensile and then compressive modulus changes. We concluded that a minimum 10 minutes recovery period is required for all three loading modes while tension and torsion would require 30 minutes recovery time to obtain an accurate measure of the damage state and recovery effect because more than $90 \%$ of strain recovery and modulus recovery were observed during this period.
\end{abstract}

Keywords : Cortical bone, Viscoelasticity, Damage, Mechanical properties, Tension, Compression, Torsion

\section{Introduction}

Skeletal fragility is an important orthopedic concern because of increased risk of fracture, and damage accumulation is one of critical component of the fracture process in bone under monotonic, creep and fatigue load conditions (Burr et al., 1997, Burr et al., 1998, Carter and Hayes, 1997, Currey, 1965, Fondrk et al., 1997, Fondrk et al., 1998, Jepsen and Davy, 1997, Jepsen et al., 1999, Knott et al., 2000, Lakes and Katz, 1979, Zioupos and Currey, 1998). Damages in bone, often recognized by micro-scale linear microcracks (Zioupos and Currey, 1994; Schaffler et al., 1995; Norman et al., 1998) and diffuse damage (Schaffler et al., 1994; Vashishth et al., 2000), have been recognized as a normal phenomenon with aging and with increased loading. Traditionally, modulus and strength degradation have been used as mechanical evidence of damage accumulation although change in modulus and strength provided limited insight into the damage process in bone (Burr et al., 1998, Carter and Hayes, 1997, Jepsen and Davy, 1997, Zioupos and Currey, 1998, Schaffler et al., 1995, Norman et al., 1998, Schaffler et al., 1994, Vashishth et al., 2000). However, creep or low-cycle fatigue studies also show that viscous properties, changes in the rate, and magnitude of relaxation are more sensitive to damage than modulus/strength (Jepsen and Davy, 1997; Martin, 2003; Fondrk et al., 1988; Fondrk et al., 1999; Currey, 1965; Knott et al, 2000; Lakes and Katz, 1979). Therefore, understanding about damage in bone is incomplete and additional experimental methods is required to observe damage accumulation and to evaluate effect of damage on mechanical properties on bone.

In a previous study, we investigated the effect of test condition and recovery time on the apparent effect of tensile damage (Joo et al., 2002, Joo et al., 2007). The results from our previous studies demonstrated that both parameters could alter measures of mechanical properties of damaged cortical bone in tension, thus leading to different estimates of the actual damage effects. Because effects of recovery time were observed for tensile material properties, it raised the question of whether such an effect might be seen for other loading modes, such as compression and torsion. It is known that the damage morphology is different for different loading modes (Burr et al., 1997, Zioupos and Currey, 1998, Akkus 
et al., 2003, Choi and Goldstein , 1992, Jepsen et al., 2001). In particular, compressive loading along the bone axis produces microdamage in the form of oblique micro-cracks, and torsional loading about the long axis produces microdamage in the form of inter-lamellar and cement line delamination. This particular difference in microscopic damage types may result different measures of material properties degradation after damage loading.

To investigate the possible differences in recovery of material properties with respect to different modes of loading and lengths of recovery time, a second study with revised testing procedure from the previous study was conducted. In the present study, we examined whether the length of the recovery period (1, 10, 30, 60 and 100 minutes) after damage and the different mode of damage loading (tension, compression or torsion) would influence the apparent measures of damage on human cortical bone in terms of changes of stiffness and viscous properties in same modes. We hypothesized that degraded material properties from damage could recover mostly within 100 minutes recovery time and similar patterns could be observed in all three loading modes (tension, compression or torsion).

\section{Materials and Methods}

Two femoral diaphysis from two female human subjects (42 and 46 years old) with no known skeletal pathologies were used for this study. The protocol for this study was approved from the Case Western Reserve University Institutional Review Board. The femur was cut into a rough, rectangular block shape using a precision band saw, and then machined into a cylindrical shape using a custom lathe. Specimens were cylindrical in shape with have a $5 \mathrm{~mm}$ grip diameter, a $4 \mathrm{~mm}$ gauge diameter, and a $16 \mathrm{~mm}$ gauge length (Fig. 1). The long axis of the specimen was parallel to the longitudinal axis of the femur and the anatomical origin of each specimen was recorded. During the sample machining, the machining condition was controlled to minimize any excessive damage on the surfaces of the sample. During the machining, each sample was visually inspected for any defects on the surface such as a harversian/volkmann canal, cracks, or machining error. A total of 36 experimental specimens were used for the study, and 12 samples were randomly assigned ( $\mathrm{n}=9$ for test group, $\mathrm{n}=3$ for control group) for tension(T), compression(C) or torsion(R) mode.

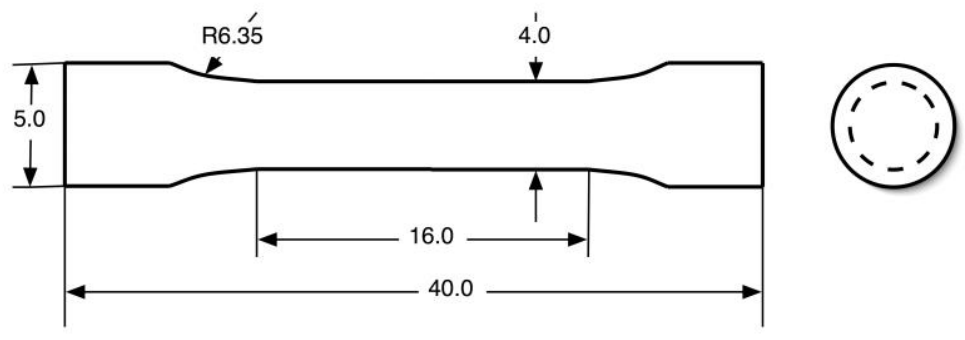

Fig. 1 The schematic diagram of specimen. All dimensions are in millimeters. The long axis of the specimen was parallel to the longitudinal axis of the femur.

Mechanical tests were conducted using servo-hydraulic testing machine (Instron, model 8501, Instron Corp., Canton, MA). Testing was performed in an environment chamber at $37^{\circ} \mathrm{C}$, and the specimen was kept wet by a $37^{\circ} \mathrm{C}$ saline solution drip during the testing. An extensometer (model 2620-530, Instron Corp., Canton, MA) was used to measure axial strain, and torsion was measured using the built-in RVDT. Wavemaker software (Instron, Instron Corp., Canton, MA) was used for test control and data acquisition.

Each specimen was loaded in one of three loading modes (Tension, Compression, or Torsion) with a sevencycle loading protocol. This protocol was designed to induce damage within machined cortical bone specimen and measure the change of mechanical properties during 100 minutes of recovery time after damage cycle, and compare it to pre-damage measures (Fig.2). 
Detail of each cycle is described in the following.

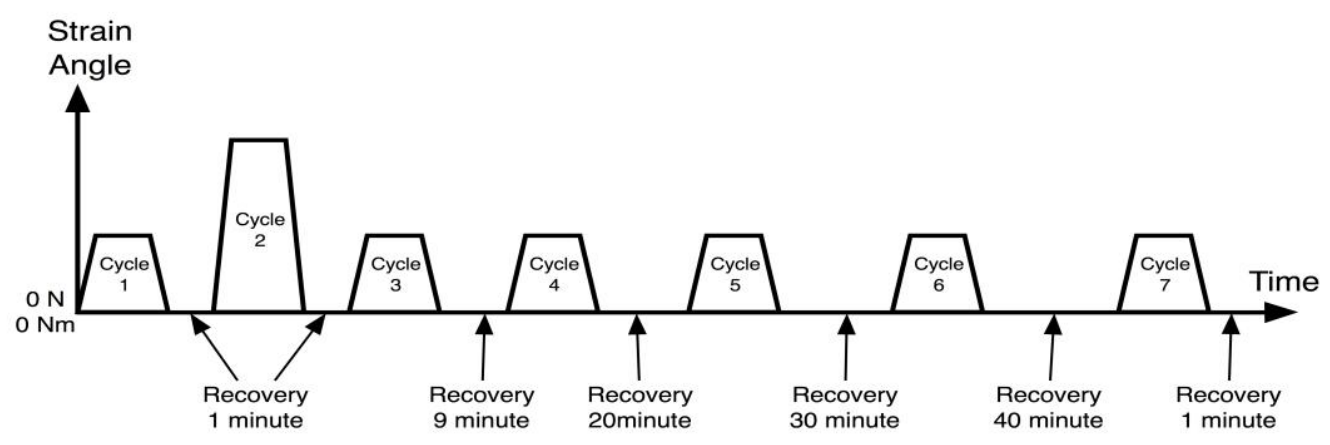

Fig. 2 Seven-cycle loading protocol was used to induce damage within machined cortical bone specimen and measure the change of mechanical properties. Each cycle is one of tension, compression or torsional mode. Compressive cycles showed in positive for convenience.

Cycle 1 - Pre-damage diagnostic cycle: the specimen was held at $0.25 \%$ strain( $(\mathrm{T}),-0.25 \% \operatorname{strain}(\mathrm{C})$ or $4.5^{\circ}$ twist $(\mathrm{R})$ for 30 seconds, unloaded to zero load or zero torque

Recovery 1: Hold specimen at zero load(T and C) or torque(R) for 1 minute.

Cycle 2 - Damage cycle: the specimen was held at $0.88 \%$ strain( $(\mathrm{T}),-0.88 \% \operatorname{strain}(\mathrm{C})$ or $18^{\circ}$ twist $(\mathrm{R})$ and held for 30 seconds to induce damage, unloaded to zero load or torque

Recovery 2: Hold specimen at zero load(T and C) or torque(R) for 1 minute.

Cycle 3 - First post-damage diagnostic cycle: the specimen was held at $0.25 \% \operatorname{strain}(\mathrm{T}),-0.25 \% \operatorname{strain}(\mathrm{C})$ or $4.5^{\circ}$ twist $(\mathrm{R})$ for 30 seconds, and unloaded to zero load(T and $\mathrm{C}$ ) or zero torque(R).

Recovery 3: Hold specimen at zero load(T and C) or torque(R) for 9 minutes.

Cycle 4 - Second post-damage diagnostic cycle, same as Cycle 3

Recovery 4: Hold specimen at zero load(T and C) or torque(R) for 20 minutes.

Cycle 5 - Third post-damage diagnostic cycle, same as Cycle 3

Recovery 5: Hold specimen at zero load(T and C) or torque(R) for 30 minutes.

Cycle 6 - Fourth post-damage diagnostic cycle, same as Cycle 3

Recovery 6: Hold specimen at zero load(T and C) or torque(R) for 40 minutes.

Cycle 7 - Fifth post-damage diagnostic cycle, same as Cycle 3

Recovery 7: Hold specimen at zero $\operatorname{load}(\mathrm{T}$ and $\mathrm{C})$ or torque(R) for 1 minute.

In the previous studies, a significant difference between zero-stress condition and zero-strain condition during recovery was observed. However, zero-stress condition caused the residual strain in the specimen, and this could alter the measurement of material properties during the recovery. Therefore, zero-stress condition was used during 100 recovery periods rather than zero-strain condition in this study. In addition, two more post-damage diagnostic cycles were added (30 $\mathrm{min}$ and $60 \mathrm{~min}$ ) to capture more insight of properties changes, in addition to post-damage diagnostic cycles (1, 10 and $100 \mathrm{~min}$ ) in previous studies (Joo et al., 2002, Joo et al., 2007).

The total recovery time (100 minutes) and the strain magnitudes of tension and compression cycles were determined based on the previous study (Joo et al., 2007), and the strain magnitude of torsion was chosen based on previous research by Jepsen and Davy, 1997. 1-minute recovery period at the end of protocol, and Recovery 7 cycle were added to compare the recovery during Recovery 1 cycle.

To examine the effects of pre and post-damage diagnostic cycles, control group testing was performed. In the three control groups for tension, compression and torsion loading mode, the damage cycle was replaced with one of diagnostic cycles $\left(0.25 \%\right.$ strain, $-0.25 \%$ strain or $4.5^{\circ}$ twist $)$ to test for the effect of fatigue by series of diagnostic cycles and diagnostic cycles associated with mechanical property degradation. Through the whole testing, loading rates and unloading rates were $1 \%$ strain/s or 30 degrees/s.

$\operatorname{Torque}(T)$-twist $(\theta)$ curves were converted to shear $\operatorname{stress}(\tau)$-strain $(\gamma)$ curves using a non-linear, timeindependent model (Nadai, 1950):

$$
\gamma=\theta a / l, \quad \tau=[\theta d T / d \theta+3 T] / 2 \pi a^{3}
$$


where $a$ is specimen radius, $l$ is gauge length and $\tau, \gamma$ are the calculated stress and strain at the outer radius of specimen.

For each diagnostic cycle and recovery period, we calculated loading modulus, secant modulus, unloading modulus, stress relaxation and strain recovery (Fig. 3). Loading modulus was defined as the slope of stress-strain curve during first $0.1 \%$ strain or 1.5 -degree rotation of each diagnostic cycle. Secant modulus was defined as the slope of stressstrain curve between the beginning of loading and the beginning of hold. Unloading tangent modulus was defined as the slope of stress-strain curve during last $0.1 \%$ strain or last 1.5 -degree rotations of each cycle. For each diagnostic cycle, we also measured stress relaxation as a reduction in load or torque during the 30-second hold period (Fig. 3) and strain recovery during 100 minute recovery time after damage cycle was measured.
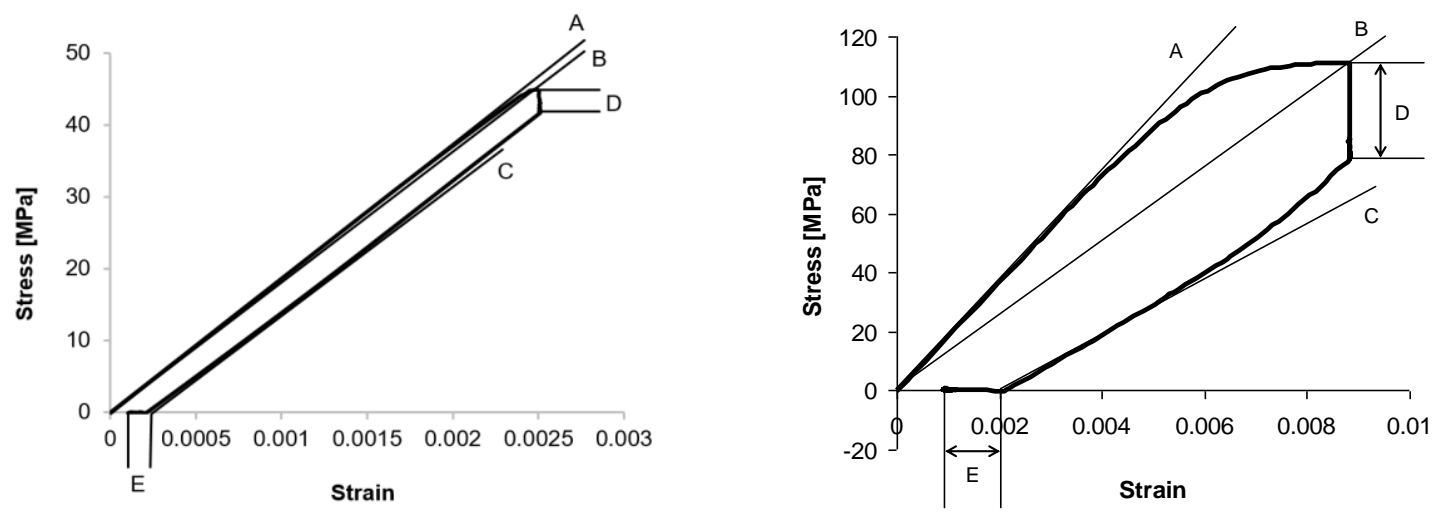

Figure 3. Stress-strain curve for the typical pre- and post-diagnostic cycle (left) and the damage cycles (right) with zero-stress hold condition. Thin lines represent measuring of mechanical properties; Loading modulus (slope of A), Secant modulus (slope of B) and Unloading modulus (slope of C), Stress relaxation (D), Strain recovery $(\mathrm{E})$. Time duration for strain recovery $(\mathrm{E})$ varied depends on the specific cycles from 1 minute to 40 minutes (Figure 2). Total summation of strain recovery time after the damage cycle was 100 minutes, and five post-damage diagnostic cycles were conducted during that period (at 1, 10, 30, 60 and $100 \mathrm{~min}$ ).

The changes in mechanical properties following damage induction were defined as $R=\left(P_{\text {Post-damage }}-P_{\text {pre }}\right.$

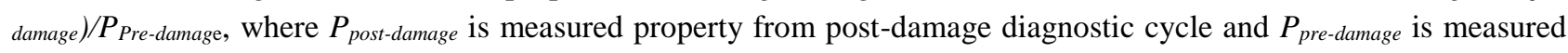
property from pre-damage diagnostic cycle. For the loading, secant and unloading modulus degradation, the parameter, $D$, was defined as $D=-R . D$ is referred to as a damage parameter, because it corresponds to simple parameter so identified for modulus decrease in other studies (Jepsen and Davy, 1997, Zioupos and currey, 1998, Akkus et al., 2003, Choi and Goldstein, 1992, Jepsen et al., 2001, Kotha and Guzelsu, 2003). The parameter $D$ normally increased from zero for

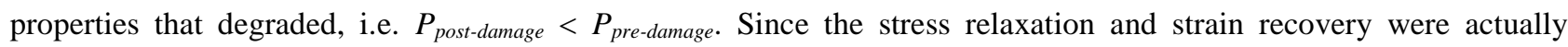
increased, this would lead to negative values in $D$. Therefore, $R$ is reported for stress relaxation $\left(R_{r e l}\right)$ and strain recovery $\left(R_{\text {rec-strain }}\right)$ while $D$ is reported for loading $\left(D_{L}\right)$, secant $\left(D_{S}\right)$ and unloading modulus $\left(D_{U}\right)$.

The effects of recovery times in different loading modes on the material properties were investigated statistically using two-tailed t-tests with Bonferroni correction $(p<0.005)$ and 2-way ANOVA's.

\section{Results}

Results of the control groups showed no significant property degradation due to the repeated diagnostic cycles. For all loading modes, average property degradation of last five diagnostic cycles by damage accumulation was negligible for any measures.

Table 1. Loading modulus change of control group in each loading mode. Average \pm S.D

\begin{tabular}{|c|c|c|c|}
\hline & Tension[GPa] & Compression[GPa] & Torsion[GPa] \\
\hline Cycle 1 & $15.90 \pm 0.48$ & $17.16 \pm 1.19$ & $4.78 \pm 0.09$ \\
\hline Cycle 2 & $15.88 \pm 0.41$ & $17.07 \pm 1.40$ & $4.69 \pm 0.08$ \\
\hline Cycle 3 & $15.86 \pm 0.39$ & $17.11 \pm 1.21$ & $4.70 \pm 0.06$ \\
\hline Cycle 4 & $15.82 \pm 0.38$ & $17.08 \pm 1.30$ & $4.72 \pm 0.06$ \\
\hline Cycle 5 & $15.79 \pm 0.34$ & $17.10 \pm 1.30$ & $4.72 \pm 0.08$ \\
\hline Cycle 6 & $15.85 \pm 0.36$ & $17.15 \pm 1.21$ & $4.73 \pm 0.06$ \\
\hline Cycle 7 & $15.82 \pm 0.33$ & $17.10 \pm 1.27$ & $4.73 \pm 0.07$ \\
\hline
\end{tabular}


In loading modulus degradation, at most, $0.32 \pm 0.18 \%$ for tension $(p=0.47), 0.29 \pm 0.11 \%$ for compression $(p=0.50)$ and $0.41 \pm 0.13 \%$ for torsional loading mode $(p=0.42)$ were measured (Table 1). Other measured material properties (secant and unloading modulus, stress relaxation) also showed no significant differences in all three loading modes due to the repeated diagnostic cycles.

\section{1 Recovery time effects}

Results for the damage groups showed that measures of damage varied significantly with recovery time and load modes (Table 2).

Table 2: Measures of mechanical properties at each cycle. Average \pm S.D.

\begin{tabular}{|c|c|c|c|c|}
\hline & & Tension & Compression & Torsion \\
\hline \multirow{4}{*}{ Cycle 1} & Loading modulus[GPa] & $18.61 \pm 1.052$ & $17.20 \pm 1.440$ & $7.412 \pm 0.626$ \\
\hline & Secant modulus [GPa] & $18.00 \pm 1.052$ & $17.03 \pm 1.442$ & $7.237 \pm 0.648$ \\
\hline & Unloading modulus[GPa] & $18.29 \pm 1.170$ & $16.92 \pm 1.481$ & $7.234 \pm 0.634$ \\
\hline & Stress relaxation[MPa] & $3.449 \pm 1.086$ & $-2.294 \pm 0.239$ & $4.545 \pm 0.519$ \\
\hline \multirow{4}{*}{ Cycle 2} & Loading modulus[GPa] & $18.56 \pm 1.082$ & $17.20 \pm 1.430$ & $7.345 \pm 0.637$ \\
\hline & Secant modulus[GPa] & $13.57 \pm 1.009$ & $14.90 \pm 1.537$ & $4.478 \pm 0.465$ \\
\hline & Unloading modulus[GPa] & $11.90 \pm 0.799$ & $12.77 \pm 1.515$ & $3.387 \pm 0.333$ \\
\hline & Stress relaxation[MPa] & $32.34 \pm 2.381$ & $-23.93 \pm 4.340$ & $25.88 \pm 2.757$ \\
\hline \multirow{4}{*}{ Cycle 3} & Loading modulus[GPa] & $17.79 \pm 1.000$ & $16.65 \pm 1.425$ & $6.486 \pm 0.569$ \\
\hline & Secant modulus[GPa] & $16.67 \pm 1.488$ & $16.27 \pm 1.481$ & $6.041 \pm 0.514$ \\
\hline & Unloading modulus[GPa] & $16.50 \pm 1.010$ & $16.01 \pm 1.498$ & $6.188 \pm 0.531$ \\
\hline & Stress relaxation[MPa] & $4.375 \pm 1.036$ & $-2.781 \pm 0.442$ & $5.076 \pm 0.475$ \\
\hline \multirow{4}{*}{ Cycle 4} & Loading modulus[GPa] & $18.00 \pm 1.055$ & $16.76 \pm 1.431$ & $6.600 \pm 0.537$ \\
\hline & Secant modulus[GPa] & $17.08 \pm 1.493$ & $16.44 \pm 1.447$ & $6.156 \pm 0.510$ \\
\hline & Unloading modulus[GPa] & $16.82 \pm 0.961$ & $16.11 \pm 1.535$ & $6.245 \pm 0.502$ \\
\hline & Stress relaxation[MPa] & $4.232 \pm 1.173$ & $-2.806 \pm 0.341$ & $4.930 \pm 0.528$ \\
\hline \multirow{4}{*}{ Cycle 5} & Loading modulus[GPa] & $18.03 \pm 0.977$ & $16.81 \pm 1.432$ & $6.674 \pm 0.536$ \\
\hline & Secant modulus[GPa] & $17.15 \pm 1.496$ & $16.47 \pm 1.447$ & $6.192 \pm 0.521$ \\
\hline & Unloading modulus[GPa] & $16.86 \pm 1.006$ & $16.15 \pm 1.484$ & $6.274 \pm 0.524$ \\
\hline & Stress relaxation[MPa] & $4.219 \pm 1.173$ & $-2.788 \pm 0.259$ & $4.772 \pm 0.857$ \\
\hline \multirow{4}{*}{ Cycle 6} & Loading modulus[GPa] & $18.08 \pm 1.034$ & $16.82 \pm 1.464$ & $6.771 \pm 0.503$ \\
\hline & Secant modulus[GPa] & $17.21 \pm 1.488$ & $16.49 \pm 1.467$ & $6.218 \pm 0.544$ \\
\hline & Unloading modulus[GPa] & $16.92 \pm 0.988$ & $16.17 \pm 1.479$ & $6.325 \pm 0.492$ \\
\hline & Stress relaxation[MPa] & $4.241 \pm 1.109$ & $-2.606 \pm 0.223$ & $4.576 \pm 0.963$ \\
\hline \multirow{4}{*}{ Cycle 7} & Loading modulus[GPa] & $18.14 \pm 1.062$ & $16.96 \pm 1.497$ & $6.839 \pm 0.489$ \\
\hline & Secant modulus[GPa] & $17.25 \pm 1.500$ & $16.51 \pm 1.483$ & $6.218 \pm 0.540$ \\
\hline & Unloading modulus[GPa] & $16.98 \pm 1.051$ & $16.21 \pm 1.510$ & $6.381 \pm 0.473$ \\
\hline & Stress relaxation[MPa] & $4.450 \pm 1.048$ & $-2.659 \pm 0.264$ & $4.067 \pm 0.770$ \\
\hline
\end{tabular}

Typically, significant changes in property ratios occurred in the first 10 minutes for loading modulus measures in all loading modes. However, longer-term trends of recovery in loading modulus ratio and unloading modulus ratio were observed for torsion (Fig. 4, 5 and 6). No long-term trends were found for stress relaxation ratio in any loading modes (Fig. 7). For all measures and recovery times, tension caused more degradation than compression for the same magnitude of strain, although torsion induced the greatest degradation. 


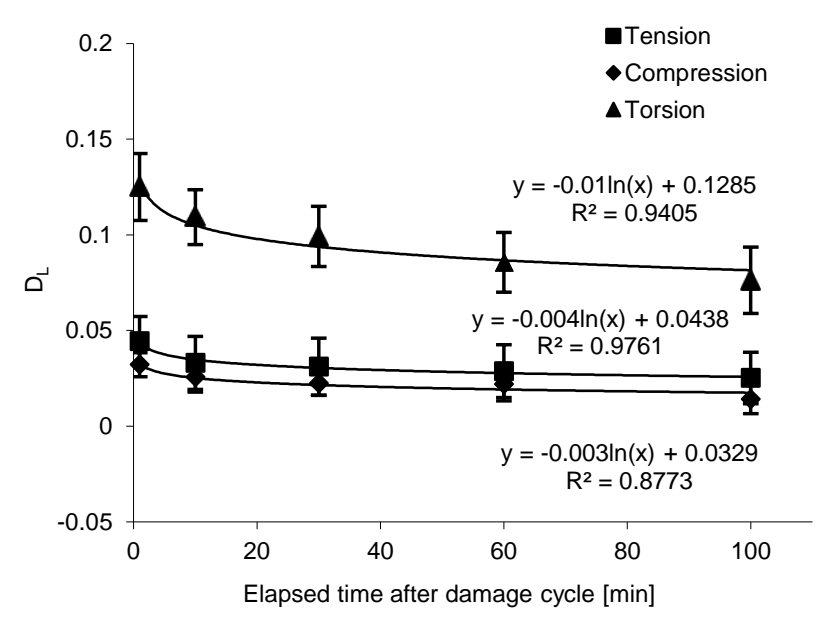

Fig. 4. Plot of loading modulus degradation $\mathrm{D}_{\mathrm{L}}=$ $\left[\left(E_{L}\right)_{\text {pre }}-\left(E_{L}\right)_{\text {post }}\right] /\left(E_{L}\right)_{\text {pre. }}$ Logarithmic fitted curves with equation and $\mathrm{R}^{2}$ are shown

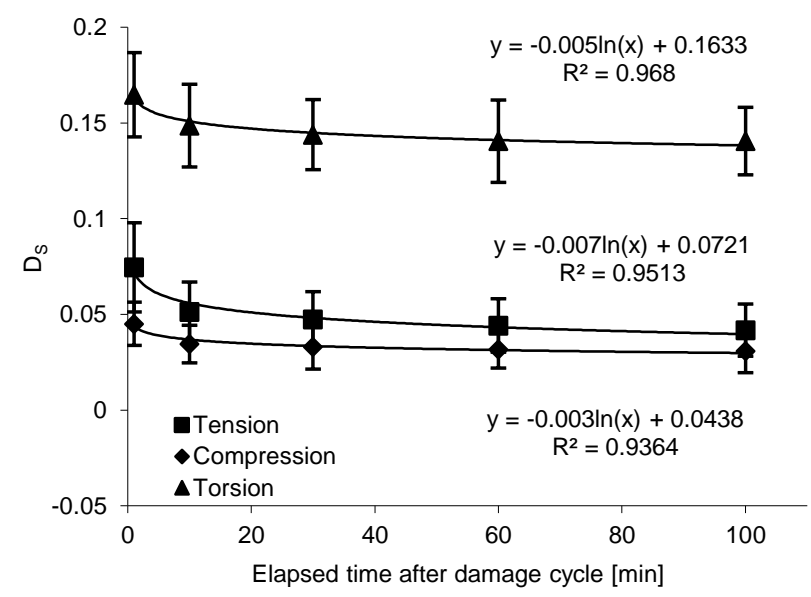

Fig. 5. Plot of secant modulus degradation $\mathrm{D}_{\mathrm{S}}=$ $\left[\left(E_{S}\right)_{\text {pre }}-\left(E_{S}\right)_{\text {post }}\right] /\left(E_{S}\right)_{\text {pre. }}$ Logarithmic fitted curves with equation and $\mathrm{R}^{2}$ are shown

Loading modulus ratios for tensile loading showed significant differences at 1 min versus 10/30/60/100 minutes, 10 minutes versus 100 minutes, and 30 minutes versus 100 minutes. Compressive loading showed significant difference at 1 minutes versus 30/60/100 minutes and 10 minutes versus 100 minutes. Loading modulus ratios for torsional loading were significantly different at 1 minutes versus 10/30/60/100 minutes, 10 minutes versus 30/60/100 minutes, and 30 minutes versus 60/100 minutes (Fig. 4).

Secant modulus ratio for tensile loading showed significant difference at 1 min versus 10/30/60/100 minutes, 10 minutes versus 60/100 minutes, and 30 minutes versus 100 minutes. Secant modulus ratio for compressive loading showed significant difference at 1 min versus 10/30/60/100 minutes and 10 minutes versus 60 minutes. Secant modulus ratio for torsional loading showed significant difference at 1 min versus 10/30/60/100 minutes (Fig. 5).

Unloading modulus ratios for tensile loading showed significant difference at 1 minute versus 10/30/50/100 minutes, 10 minutes versus 60/100 minutes, and 30 minutes versus 100 minutes. Unloading modulus ratios for compressive loading showed significant difference at 1 minute versus 10/30/50/100 minutes, while torsional loading showed significant difference at 1 minute versus 100 minutes, and 10 minutes versus 100 minutes (Fig. 6). Stress relaxation showed no significant differences for all recovery times in any loading modes (Fig. 7).

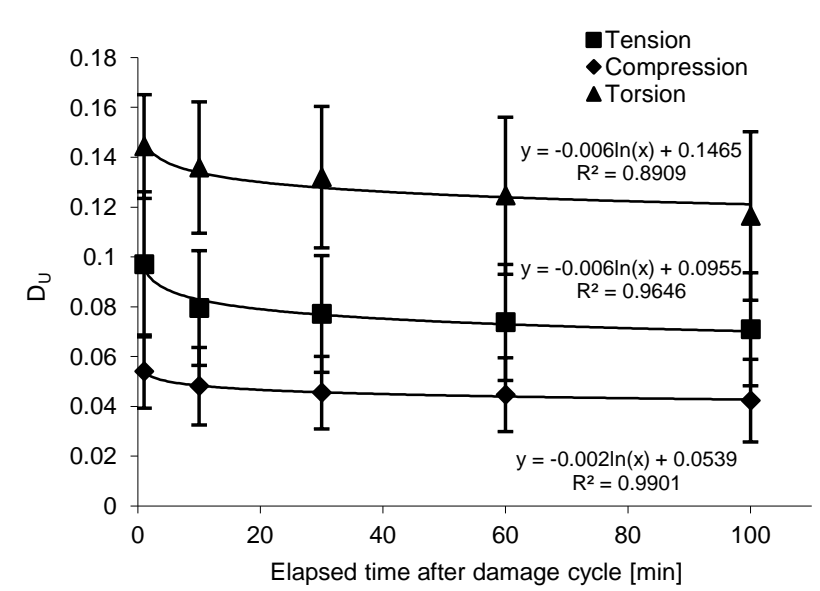

Fig. 6. Plot of unloading modulus degradation $\mathrm{D}_{\mathrm{U}}$ $=\left[\left(\mathrm{E}_{\mathrm{U}}\right)_{\text {pre }}-\left(\mathrm{E}_{\mathrm{U}}\right)_{\text {post }}\right] /\left(\mathrm{E}_{\mathrm{U}}\right)_{\text {pre. }}$. Logarithmic fitted curves with equation and $\mathrm{R}^{2}$ are shown

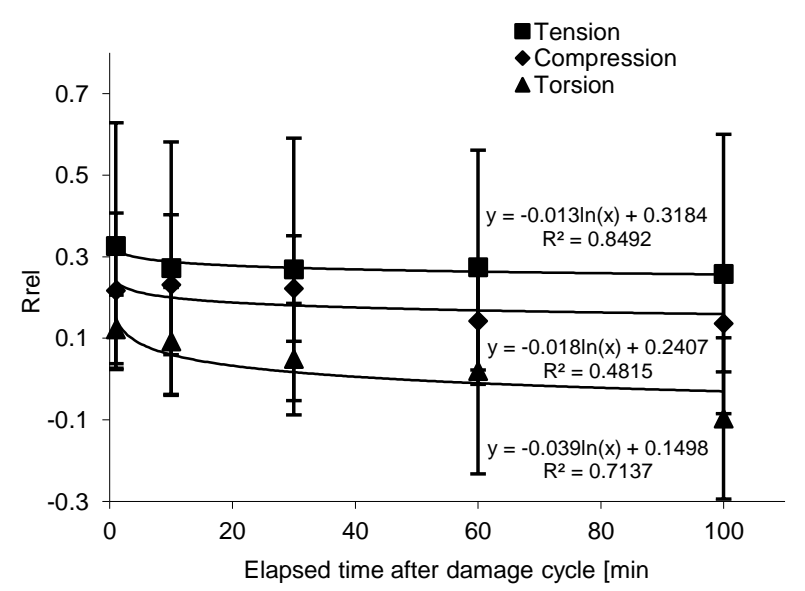

Fig. 7. Plot of stress relaxation change during $30 \mathrm{sec}$ hold parameter $R_{\text {rel }}=\left[\left(\sigma_{\text {rel }}\right)_{\text {post }}-\left(\sigma_{\text {rel }}\right)_{\text {pre }}\right] /\left(\sigma_{\text {rel }}\right)_{\text {pre }}$ Logarithmic fitted curves with equation and $\mathrm{R}^{2}$ are shown 
Strain recovery in all modes showed significant differences between 1 and the other recovery times, 10 and the other recovery times (Fig. 8). In addition, strain recovery showed significant change $(p<0.005)$ between 1 minute vs. 10 minutes in all loading modes, and between 10 minutes vs. 30 minutes in tension and torsion. After 30 minutes of recovery time, strain recovery in compression and torsion showed no significant recovery but tension did between 30 minutes vs. 60 minutes (Fig. 8).

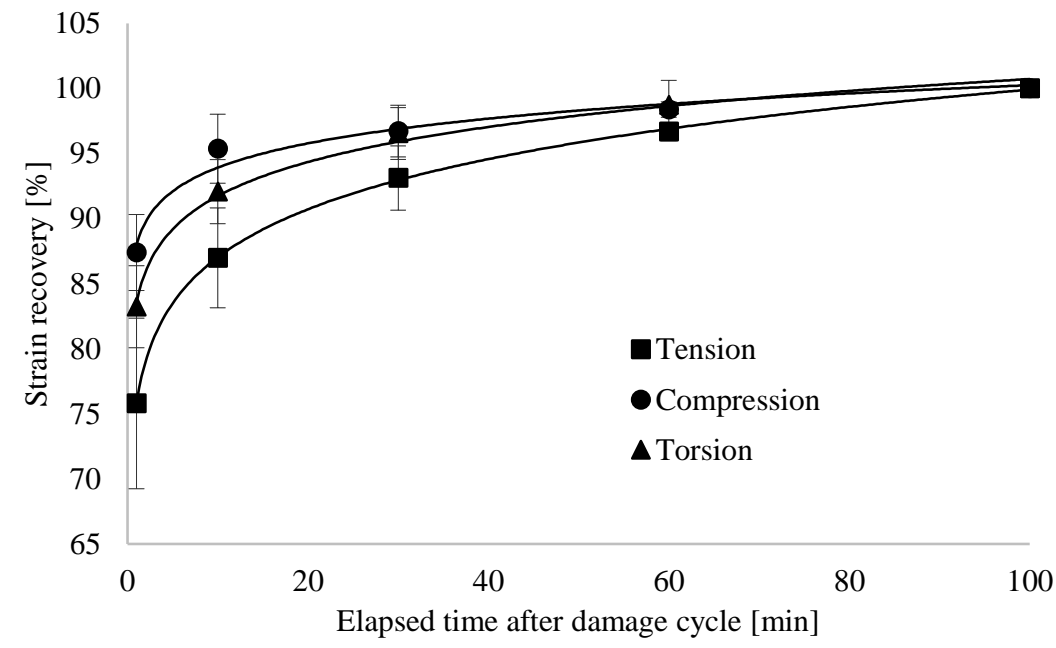

Fig. 8. Histories of strain recovery during 100 minutes recovery period. Note the every data were normalized to its maximum recovery amount at 100 minutes respectively. Recoveries of strain in compression and torsion were similar to each other after 30 minutes of recovery time although strain tension showed slower recovery. All values are mean \pm SD. The curve fitting showed $y=75.797 \mathrm{x}^{0.06}$ and $\mathrm{R}^{2}=0.9998$ for tension, $\mathrm{y}=87.988 \mathrm{x}^{0.0283}$ and $\mathrm{R}^{2}=0.9719$ for compression; $\mathrm{y}=83.531 \mathrm{x}^{0.0406}$ and $\mathrm{R}^{2}=0.9945$ for torsion. Note $\mathrm{x}$ and $\mathrm{y}$ correspond to elapsed time after damage and strain recovery.

\subsection{Loading mode effects}

With respect to loading mode effects, all torsional modulus changes were largest followed by tensile and then compressive modulus changes. Loading modulus ratio and secant modulus ratio showed significant differences between tension and torsion, and between compression and torsion (Fig. 4 and 5). Unloading modulus ratios showed differences in tension versus compression, tension versus torsion and compression versus torsion (Figure 6). Stress relaxation ratio showed no significant differences between any modes (Fig. 7). Average strain recovery rates between 1 and 10 minutes were not significantly different among loading modes. Recovery rate in compression was different from recovery rate in tension and torsion between 10 and 30 minutes (Fig. 8).

\section{Discussion}

In this study, we have investigated the effects of recovery times $(1,10,30,60$ and 100 minutes) on common damage measures in three commonly used loading modes (tension, compression and torsion). In a previous study, recovery time and recovery conditions had been found to affect tensile damage measures (Joo et al, 2002). The first goal of the current study was to see how the effects of recovery time varied with respect to loading mode. We found consistent recovery effects across the three modes, although the details varied. With respect to the second goal, we found that the most strongly affected measures were stress/torque relaxation during the diagnostic cycle. This behaviour had been observed in previous studies of tensile damage and torsional damage on viscoelastic properties (Jepsen and Davy, 1997, Joo et al, 2002, Knothe and Knothe, 2000). With respect to the third goal, a nominal 10 minute recovery proved to be a reasonable time for stabilization of most measures. As previously noted for tensile damage (Joo et al, 2002), we also found that load (stress or torque) relaxation effect was the most quickly stabilized, showing no differences in any recovery time from 3 minutes on. However, torsional stiffness properties tended to show recovery effects over longer times than either axial loading mode.

In this study, testing samples were held at zero stress or zero torque during recovery times. In our previous study of tensile damage recovery effects, zero strain or zero degree holding condition during recovery times caused residual compressive stresses in the specimens (Joo et al, 2002). The corresponding damage measures were less than those for 
zero stress or torque holding condition were. Whether this represents a true damage recovery effect is open to question, but we chose to minimize the effect of residual stresses or torques by using the zero stress or torque recovery condition in this study.

In addition, the effect of fatigue by series of diagnostic cycles was not observed in this study by control group testing. Since less than $0.4 \%$ of modulus (maximum degradation in loading modulus) measures were degraded during control group testing, property degradation reflected an effect of damage accumulation was induced by only a damage cycle during the test.

Recovery ratio curve of measured modulus showed that effect of damage accumulation in bone was decreased with increased recovery time for all loading modes. However, recovery times beyond 10 minutes were not influential for most measures although some modulus properties were still evident after 10 minutes in torsion. During this period, approximately $90 \%$ of total recovery of 100 minutes was observed.

Damage effects varied with loading modes. At the same strain level, modulus reductions were greater in tension than compression. These results are consistent with our previous study and other studies that bone is weaker in tension than compression because microdamage will accumulate more rapidly in tension than compression (Reilly and Burstein, 1975, Reilly and Currey, 1998).

It is possible to compare Torsional strain level with linear strain level using the concept of equivalent strain. We have previously used the equivalent strain equation to estimate the effective strain state where $\varepsilon$ is linear strain and $\gamma$ is torsional strain (Cezayirlioglu et al, 1985).

$$
\varepsilon_{e q}=\sqrt{\varepsilon^{2}+\frac{1}{3} \gamma^{2}}
$$

Using this equation, the equivalent strain at the outer surface for the torsional samples was about $2.25 \%$ equivalent strain, compared to $0.88 \%$ for the axial strains. This difference of damage strain level could explain the greater degradation of torsional stiffness, and different recovery time effect compared to tension and compression group. We also note that the strain state for torsion is non-homogeneous, varying from a maximum at the outer surface to zero at the center of the specimen. This means that comparison of axial (tension and compression) and torsional test are primarily qualitative. However, the fundamental finding that after 10 minutes there are only small changes in degradation measures, holds for all three modes.

The differences in results with respect to damage mode may also reflect qualitative differences in damage morphology. The inter-lamellar longitudinal cracks are due to torque-generated shear stress and are typical for anisotropic materials, like bone, which are weaker in the longitudinal plane than the transverse plane (Carter and Hayes, 1997, Currey and Brear, 1974). Jepsen et al. showed that the lamellar interface in bone is weak and is the principal site of shear damage formation but the lamellar interface was shown to be highly effective in keeping cracks isolated from each other. This result is consistent with other research suggesting that torsional loading plays a very important role in determining the fatigue life of cortical bone in vivo (Jepsen and Davy, 1997, Jepsen et al, 1999, Pattin et al, 1996, Cezayirlioglu et al., 1985, Martin, 2003, Yeni and Fyhrie, 2002).

The results showed that the change of stress relaxation in all modes does not depend on the recovery time after loading. In addition, the magnitudes of the changes in stress relaxation tend to be greater, especially compared to the loading modulus. The loading modulus is most often used as a simple damage measure, although the secant modulus is often used for cyclic loading studies. The reason for the greater change in viscous properties is unknown at this point. Previous studies showed that microcracking is the main form of damage accumulation in bone and is closely associated with stiffness loss (Burr et al, 1998, Zioupos and Currey, 1998, Norman et al., 1998, Kotha and Guzelsu, 2003, Martin, 2003, Zioupos and Currey, 1994). Other studies showed that even at stiffness reductions so low that producing a calculated damage estimate of $0 \%$, tiny (order of $10 \mu \mathrm{m}$ in length) tensile microcracks were visible. These microcracks could be one of reason for large viscoelastic properties changes in damaged bone. An explicit calculation of the contribution of fluid motion to the viscoelastic response of porous materials has been studied by Rusch (Rusch, 1965). In that study, the results showed that contribution of fluid flow to the loss of tangent of cortical bone is critical. This argument depends on the assumption that a volume change occurs in the solid bone.

For the case of torsion, there is no overall volume change and no energy dissipation due to this type of fluid flow. Local volume change due to inhomogeneity of bone may cause some volume change but this would be very small and essentially negligible. 
The interfacial motion (cement line) and molecular properties of collagen are another source of viscoelastic property changes in damaged cortical bone. Studies of nanoscale damage mechanisms showed that increase of collagen length results viscoelastic properties changes in connective tissue made of type I collagen, and the decrease of diameter of the collagen microfibrils increased viseoelastic properties and changed elastic modulus (Christiasen et al, 2000, Jager and Fratzl, 2000).

In this study, the damage loading cycle caused significant damage on both bone collagen microfibrils and bone mineral structures. While damage in bone mineral structure in the form of microcracks cannot be recovered in 100 minutes, some damages, not all, in collagen microfibrils can be reversed (Christiasen et al, 2000, Jager and Fratzl, 2000 ). The effects of damage in bone were observed even at the point of 100 minutes after damage cycle while the measure of damage was still decreasing. This residual damage at 100 min might indicate an amount of non-recovered damage in bone structures. This result can explain the effect of recovery time on mechanical properties of damaged cortical bone, and can be used to develop a viscous material model with an effect of damage

Several limitations to this study must be recognized. In this study we treated damage was simply represented by scalar measures of change. In fact, it is complex process involving non-uniform, anisotropic damage distribution associated with each damage mode. Another limitation is that we conducted one damage loading (strain) level for each loading mode, so possible damage amplitude effects on recovery are unknown. In addition, based on an equivalent strain estimate, there was a difference of damage strain magnitude between axial tests and torsional tests and the torsional tests do not represent a homogeneous strain state. Even for axial loads, the same damage strain levels for tension and compression amount to different strains relative to the nominal yield strain. The $0.88 \%$ strain is above the nominal tensile yield strain but only approaching commonly accepted values for compressive yield strain. It is encouraging that in spite of these differences, the time course of apparent recovery is similar across the three different loading modes.

We note that five diagnostic cycles after the damage cycle were done to measure the mechanical properties changes caused by the damage. It is possible the diagnostic cycles added small amounts of residual strain because of zero loading hold condition during the cycle. However, time vs strain measures from test groups showed that the amount of residual strain has no or very small effects on the measurement of strain recovery during 100 minutes overall. The overall strain recovery behavior was similar to that in the previous study, which only had one post-damage diagnostic cycle at 100 minutes. Based on the observation, we believed that five post-damage diagnostic cycles did not alter strain recovery behavior after damage while it did allow measuring additional property changes at the certain time for comparison before and after damage

We also note that the set of recovery times that were considered here filled in the gaps between 10 minutes and 100 minutes from the previous study (Joo et al, 2002). However, the intermediate times between 1 and 10 minutes were not examined experimentally but the approximation was possible using curve fitting. Curve fitting results showed significant strain recovery in first 10 minutes in all loading modes. Therefore, the 10-minute recovery recommendation is possibly conservative for all loading modes. We note again that shorter recovery times do not invalidate the data, but mean that comparisons to data with different recovery times should not be made.

This study provided new views to examine damage accumulation in bone, relationship between damage and mechanical properties. First, the choice of damage measure is important. The change in viscous effect was bigger than modulus for all loading modes. This result indicated that damage accumulation and recovery might not only affect of strength and modulus but also affect viscous properties, and viscoelastic measures may allow for the inherent anisotropic nature of bone and the anisotropic nature of the damage. Second, the compression caused less damage than tension against same strain magnitude. Torsion induced most damage than axial modes. However, because the physical characteristics of shear damage are not known and damage magnitude by torsion was different from tension and compression, more research is needed

\section{Conclusion}

We concluded that a minimum 10 minutes recovery period is required for all three loading modes while tension and torsion would require 30 minutes recovery time to obtain an accurate measure of the damage state and recovery effect because more than $90 \%$ of strain recovery and modulus recovery were observed during this period. This study suggested that measures that were taken without allowing for any recovery are probably different from what might have been obtained if a recovery period had been allowed. 


\section{Conflict of Interest}

There is no conflict of interests regarding the publication of this paper.

\section{Acknowledgements}

This study is supported by NIH Grants AR43785 and AR44927. Tissues provided by Musculoskeletal Transplant Foundation.

\section{References}

Akkus, O., Polyakova-Akkus, A., Adar, F, Schaffler, M.B., Relationship between damage accumulation and mechanical property degradation in cortical bone: microcrack orientation is important. Journal of Biomedical Material Research, Vol. 65, No 4 (2003), pp.482-488.

Burr. D.B., Forwood, M.R., Fyhrie, D.P., Martin, B.R., Schaffler, M.B., Turner, C.H., Bone microdamage and skeletal fragility in osteoporotic and stress fractures. Journal of Bone and Mineral Research, Vol. 12, No. 1 (1997), pp.615

Burr, D.B., Turner, C.H., Naick, P., Forwood, M.R., Ambrosius, W., Hasan, M.S., Pidaparti, R., Does Microdamage Accumulation Affect the Mechanical Properties of Bone?, Journal of Biomechanics 31 (1998), pp.337-345.

Carter, D.R., Hayes, W.C., 1977. Compact bone fatigue damage - I. residual strength and stiffness. Journal of Biomechanics Vol. 10 (1997), pp.325-337.

Cezayirlioglu, H., Bahniuk, E., Davy, D.T., Heiple, K.G., Anisotropic yield behavior of bone under combined axial force and torque. Journal of Biomechanics Vol.18, No. 1 (1985), pp.61-69

Choi K., Goldstein S.A., A comparison of the fatigue behavior of human trabecular and cortical bone tissue. Journal of Biomechanics. Vol. 25, No. 12 (1992), pp.1371-1381.

Christiasen, D.L., Huang, E.K., Silver, F.H., Assembly of type-I collagen:fusion of fibril subunits and influence of fibril diameter on mechanical properties. Matrix Biology Vol. 19 (2000), pp.409-420

Currey, J.D., Anelasticity in bone and echinoderm skeletons. Journal of Experimental Biology Vol. 43 (1965), pp.297292.

Currey, J.D., Brear, K., Tensile yield in bone. Calcified Tissue Research Vol. 15 (1974), pp.173-179

Fondrk, M.T., Bahniuk, E.H., Davy, D.T., Inelastic strain accumulation in cortical bone during rapid transient tensile loading. Journal of Biomechanal Engineering Vol. 12 (1997), pp.616-621.

Fondrk, M., Bahniuk, E., Davy D.T., Michaels, C., Some viscoplastic characteristics of bovine and human cortical bone. Journal of Biomechanics Vol. 21 (1998), pp.623-630

Jager, I., Fratzl, P., Mineralized collagen fibrils: a mechanical model with a staggered arrangement of mineral particles. Biophysical Journal Vol. 79 (2000), pp.1737-1746

Jepsen, K.J., Davy, D.T., Comparison of damage Accumulation Measures in Human Cortical Bone. Journal of Biomechanics Vol. 9 (1997), pp.891-894.

Jepsen, K.J., Davy, D.T., Akkus, O., Observations of damage in bone. Bone Mechanics Handbook. (2001) CRC Press

Jepsen, K.J., Davy, D.T., Krzypow, D.J., The role of the lamellar interface during torsional yielding of human cortical bone. Journal of Biomechanics Vol. 3 (1999), pp.303-310.

Joo, W., Jepsen, K.J., Davy, D.T., Effect of recovery time and test conditions on tensile damage measures in cortical bone. In Proceedings of the $27^{\text {th }}$ Orthopaedic Research Society (2002).

Joo, W., Jepsen, K.J., Davy, D.T., The effect of recovery time and test conditions on viscoelastic measures of tensile damage in cortical bone. Journal of Biomechanics. Vol 40, No 12. (2007), pp 2731-2737

Knothe Tate, M.L., Knothe, U., An ex vivo model to study transport processes and fluid flow in loaded bone. Journal of Biomechanics Vol. 33, NO. 2 (2000), pp.247-254

Knott, D.F., Jepsen, K.J., Davy, D.T., Age related changes in tensile damage accumulation behavior of human cortical bone. In Proceedings of the $25^{\text {th }}$ Orthopaedic Research Society (2000).

Kotha S.P., Guzelsu N., Tensile damage and its effects on cortical bone. Journal of Biomechanics. Vol. 36, No. 11 (2003), pp.1683-1689. 
Lakes, R.S., Katz, J.L., Viscoelastic properties of wet cortical bone-II. Relaxation mechanisms. Journal of Biomechanics Vol. 12 (1997), pp.679-687.

Martin, R.B., Fatigue microdamage as an essential element of bone mechanics and biology. Calcified Tissue International. Vol 73, No. 2 (2003), pp.101-107

Norman, T.L., Yeni, Y.N., Brown, C.U., Wang, Z., Influence of microdamage on fracture toughness of the human femur and tibia. Bone Vol. 23 (1998), pp.303-306.

Pattin, C.A., Caler, W.E., Carter, D.R., Cyclic mechanical property degradation during fatigue loading of cortical bone. Journal of Biomechnics Vol. 29, No. 1 (1996), pp.69-79.

Reilly, D.T., Burstein, A.H., The elastic and ultimate properties of compact bone tissue. Journal of Biomechanics Vol. 8 (1975), pp.393-405

Reilly, G.C., Currey, J.D., Relationship between microcracking density and changes in mechanical properties in bone. In proceedings of the 11th Conference of the European Society of Biomechanics, Toulouse (1998)

Rusch, K., Dynamic behavior of flexible open cell forms. Ph.D. Thesis, Department of polymer Science, University of Ohio (1965)

Schaffler, M.B., Choi, K., Milgrom, C., Aging and matrix microdamage accumulation in human compact bone. Bone Vol. 17 (1995), pp.521-525.

Schaffler, M.B., Pitchford, W.C., Choi, K., Riddle, J.M., Examination of compact bone microdamage using backscattered electron microscopy. Bone Vol. 15 (1994), pp.483-488.

Vashishth, D., Koontz, J., Qiu, S.J., Lundin-Cannon, D., Yeni, Y.N., Schaffler, M.B., In vivo diffuse damage in human vertebral trabecular bone. Bone Vol. 26 (2000), pp.147- 152.

Yeni, Y.N., Fyhrie, D.P., Fatigue damage-fracture mechanics interaction in cortical bone. Bone Viol. 30, No. 3 (2002), pp.509-514

Zioupos, P., Currey, J.D., Extent of microcracking and morphology of microcracks in damaged bone. Journal of Material Science Vol. 29, No. 4 (1994), pp.978-986.

Zioupos, P., Currey, J.D., Changes in the stiffness, strength, and toughness of human cortical bone with age. Bone Vol. 22 (1998), pp.57-66. 